\title{
Physical properties and stability evaluation of fish oil-in-water emulsions stabilized using thiol- modified $\beta$-lactoglobulin fibrils-chitosan complex
}

\begin{abstract}
Fish oil-in-water emulsions containing fish oil, thiol-modified $\beta$-lactoglobulin ( $\beta$-LG) fibrils, chitosan and maltodextrin were fabricated using a high-energy method. The results showed that chitosan coating induced charge reversal; denoting successful biopolymers complexation. A significantly $(\mathrm{p}<0.05)$ larger droplet size and lower polydispersity index value, attributed to the thicker chitosan coating at the oil-water interface, were observed. At high chitosan concentrations, the cationic nature of chitosan strengthened the electrostatic repulsion between the droplets, thus conferring high oxidative stability and low turbidity loss rate to the emulsions. The apparent viscosity of emulsions stabilized using thiol-modified $\beta$-LG fibrilschitosan complex was higher than those stabilized using $\beta$-LG fibrils alone, resulting in the former's higher creaming stability. Under thermal treatments $\left(63{ }^{\circ} \mathrm{C}\right.$ and $\left.100{ }^{\circ} \mathrm{C}\right)$, emulsions stabilized using thiol-modified $\beta$-LG fibrils-chitosan complex possessed higher heat stability as indicated by the consistent droplet sizes observed. Chitosan provided a thicker protective layer that protected the oil droplets against high temperature. Bridging flocculation occurred at low chitosan concentration $(0.1 \%, \mathrm{w} / \mathrm{w})$, as revealed through microscopic observations which indicated the presence of large flocs. All in all, this work provided us with a better understanding of the application of protein fibrils-polysaccharide complex to produce stable emulsion.
\end{abstract}

Keyword: $\beta$-Lactoglobulin; Fibril; Chitosan; Fish oil emulsion; Emulsion stability; Complexation 